\title{
science \\ Open data policy of Science Editing \\ Editorial
}

Kihong Kim

Department of Physics, Ajou University, Suwon, Korea

Recently, the number of journals adopting an open data policy has been rapidly increasing. A common form of this policy requires authors to provide the raw data for the presented results and make it available in a public domain. An obvious benefit of making scientific data open is that it will enhance the transparency of the results and suppress research frauds. Furthermore, by allowing open data to be reanalyzed and reused freely without any restrictions, one can also help the verification and reinterpretation of the original results. We believe these benefits are sufficiently large and support the basic ideas of the open data movement. Therefore, we have introduced the following open data policy to Science Editing:

"For clarification on result accuracy and reproducibility of the results, raw data or analysis data will be deposited to a public repository, for example, Harvard Dataverse (https://dataverse.harvard.edu) after acceptance of the manuscript. Therefore, submission of the raw data or analysis data is mandatory. If the data is already a public one, its URL site or sources should be disclosed. If data cannot be publicized, it can be negotiated with the editor. If there are any inquiries on depositing data, authors should contact the editorial office."

This policy can be found on our web page about Best Practice (https://www.escienceediting. org/about/best_practice.php) and will be effective from the next issue (volume 6, number 1, February 2019). We hope our policy can help making the results published in Science Editing more transparent and more open.

\section{Conflict of Interest}

No potential conflict of interest relevant to this article was reported. 